ESAIM: PROCEEDINGS AND SURVEYS, 2017, Vol. 57, p. 97-103

Oana Silvia Serea \& Walter Briec Editors

\title{
ON HYPERSPACES OF MAX-PLUS AND MAX-MIN CONVEX SETS
}

\author{
LIDIYA BAZYLEVYCH ${ }^{1}$ AND MYKHAILO ZARICHNYI ${ }^{2}$
}

\begin{abstract}
The paper is devoted to some new results concerning the topology of hyperspaces of maxplus convex subsets in Euclidean spaces and some other spaces.

Résumé. L'article est dévoué à de nouveaux résultats concernant la topologie de hyperespaces de sous-ensembles max-plus convexes dans des espaces euclidiens et d'autres espaces.
\end{abstract}

\section{INTRODUCTION}

Nadler, Quinn, and Stavrakas [14] proved that the hyperspace of compact convex subsets of the Euclidean space $\mathbb{R}^{n}, n \geq 2$, is homeomorphic to the space $Q \backslash\{*\}$, where $Q$ is the Hilbert cube. This result as well as another results from [14] opened the door to applications of methods of infinite-dimensional topology to convexity.

In the recent decade, there were widely investigated the so called max-plus and max-min convex sets. They are counterparts of the convex sets in the idempotent mathematics, i.e. a part of mathematics in which (some of) the usual operations in the set of reals is replaced by some idempotent operations; see, e.g., [13]. Motivations of consideration of max-plus convex sets as well as survey of results in max-plus convexity can be found in [10].

Some recent results are devoted to the hyperspaces of the max-plus and max-min convex sets. In particular, in $[2,3]$, results analogous to the mentioned theorem of Nadler, Quinn, and Stavrakas were obtained. The main result of [4] stated that the hyperspace of max-plus convex subsets in the spaces $\mathbb{R}^{\tau}$ is homeomorphic to $\mathbb{R}^{\tau}$ if $\tau \in\left\{\omega, \omega_{1}\right\}$.

The present note is also devoted to the hyperspaces of max-plus convex sets. Some of the obtained results can be extended over the case of max-min convex sets.

The authors are indebted to the referee for helpful comments and corrections.

\section{Preliminaries}

All maps of topological spaces are assumed to be continuous. Recall that a map is open if the image of every open set is open. A map is proper if the preimages of compact subsets are compact.

By $I$ we denote the segment $[0,1]$. By $\bar{A}$ we denote the closure of a set $A$ in a topological space.

\footnotetext{
1 Department of Mechanics and Mathematics, Ivan Franko National University of Lviv, Universytetska Str. 1 , 79000 Lviv, Ukraine

2 Institute of Applied Mathematics and Fundamental Sciences, National University "Lviv Polytechnica", Mytropolyt Andrei str. 5, Building 4, Lviv, Ukraine

(c) EDP Sciences, SMAI 2017
} 


\subsection{Max-plus and max-min convexity}

Let $\mathbb{R}_{\max }=\mathbb{R} \cup\{-\infty\}$ and let $\tau$ be a cardinal number. Given $x, y \in \mathbb{R}^{\tau}$ and $\lambda \in \mathbb{R}_{\max }$, we denote by $x \oplus y$ the coordinatewise maximum of $x$ and $y$ and by $\lambda \odot x$ the vector obtained from $x$ by adding $\lambda$ to every its coordinate. Given $\lambda \in \mathbb{R}_{\max } \cup\{\infty\}$ and $x=\left(x_{\alpha}\right) \in \mathbb{R}^{\tau}$, we define $\lambda \otimes x=\left(\min \left\{\lambda, x_{\alpha}\right\}\right)$.

A subset $A$ in $\mathbb{R}^{\tau}$ is said to be max-plus convex if $\alpha \odot a \oplus \beta \odot b \in A$ for all $a, b \in A$ and $\alpha, \beta \in \mathbb{R}_{\max }$ with $\alpha \oplus \beta=0$. A subset $A$ in $\mathbb{R}^{\tau}$ is said to be max-min convex if $\alpha \otimes a \oplus b \in A$ for all $a, b \in A$ and $\alpha \in \mathbb{R}_{\max }$.

The minimal max-plus (respectively max-min) convex set containing a subset $A$ in $\mathbb{R}^{n}$ is called the max-plus convex hull (respectively max-min convex hull) of $A$ and is denoted by $h_{\mathrm{mp}}(A)\left(\operatorname{respectively} h_{\mathrm{mm}}(A)\right)$.

\subsection{Hyperspaces}

Given a topological space $X$, by $\exp X$ we denote the space of all nonempty compact subsets in $X$. The sets of the form

$$
\left\langle U_{1}, \ldots, U_{n}\right\rangle=\left\{A \in \exp X \mid A \subset \cup_{i=1}^{n} U_{i}, A \cap U_{i} \neq \emptyset \text { for all } i\right\},
$$

where $U_{1}, \ldots, U_{n}$ are open sets in $X$, comprise a base of the Vietoris topology in exp $X$. If $(X, d)$ is a metric space, then the Vietoris topology on $\exp X$ is generated by the Hausdorff metric $d_{H}$ :

$$
d_{H}(A, B)=\max \left\{\sup _{a \in A} \inf _{b \in B} d(a, b), \sup _{b \in B} \inf _{a \in A} d(a, b)\right\}
$$

where $A, B \in \exp X$.

Let $\exp _{n} X$ denote the subspace of $\exp X$ consisting of all sets of cardinality $\leq n$.

By $\operatorname{mpcc}(X)$ we denote the hyperspace of nonempty max-plus convex compact subsets in $X \subset \mathbb{R}^{n}$. Given $n \in \mathbb{N}$, let $\operatorname{mpcc}_{n}(X)$ denote the subspace of $\operatorname{mpcc}(X)$ consisting of convex hulls of the sets of cardinality $\leq n$.

\subsection{Absolute retracts and some infinite-dimensional spaces}

A metrizable space $X$ is an absolute (neighborhood) retract (briefly: AR (ANR)) if it is a retract of (an open subset of) any metrizable space containing $X$ as a closed subset. The convex subsets of normed spaces are examples of AR-spaces. See, e.g., [5] for the backgrounds of the theory of retracts.

The notion of $\mathrm{A}(\mathrm{N}) \mathrm{R}$ can be also defined in another classes of topological spaces, in particular, in the class of compact Hausdorff spaces (see [15]).

In the sequel, we will need the following notion, which was introduced in [11]: a $c$-structure on a topological space $X$ is an assignment, to every nonempty finite subset $A$ of $X$, of a contractible subspace $F(A)$ of $X$, such that $F(A) \subset F\left(A^{\prime}\right)$ whenever $A \subset A^{\prime}$. A pair $(X, F)$, where $F$ is a $c$-structure on $X$, is called a $c$-space. A subset $E$ of $X$ is called an $F$-set if $F(A) \subset E$ for any finite $A \subset E$. A metric space $(X, d)$ is said to be a metric l.c.-space if all the open balls in it are $F$-sets and all open $r$-neighborhoods of $F$-sets are also $F$-sets.

A compact metric space $X$ is said to satisfy the Disjoint Disc Property (DDP) if every map $f: I^{n} \rightarrow X$ can be approximated in the sense of uniform convergence by maps with disjoint images. The following characterization theorem for the Hilbert cube $Q=I^{\omega}$ is proved by H. Toruńczyk [16]: a compact metrizable AR-space $X$ is homeomorphic to $Q$ if and only if $X$ satisfies DDP.

A metrizable space $X$ is said to be a Hilbert manifold if $X$ is locally homeomorphic to the Hilbert space $\ell^{2}$.

Let $\mathcal{U}$ be an open cover of a topological space $X$. We say that a map $f: Y \rightarrow X$ is a $\mathcal{U}$-domination if $f$ is a proper map and there is a map $g: X \rightarrow Y$ such that $f g$ is $\mathcal{U}$-homotopic to $1_{X}$ (the latter means that there exists a homotopy $H: X \times[0,1] \rightarrow X$ of the maps $f g$ and $1_{X}$ such that, for every $x \in X$, there is $U \in \mathcal{U}$ with $H(\{x\} \times[0,1]) \subset U)$. It is known that every proper retraction onto $X$ is a $\mathcal{U}$-domination, for every open cover $\mathcal{U}$ of $X$.

The following result is proved by H. Toruńczyk [17, Theorem 4.1].

Theorem 1.1. Let $X$ be a complete $A N R$. If, for every open cover $\mathcal{U}$ of $X$, the space $X$ is $\mathcal{U}$-dominated by a Hilbert manifold, then $X$ is a Hilbert manifold itself. 
By $\mathbb{R}^{\infty}$ we denote the direct limit of the sequence

$$
\mathbb{R}^{1} \hookrightarrow \mathbb{R}^{2} \hookrightarrow \mathbb{R}^{3} \hookrightarrow \ldots
$$

By $Q^{\infty}$ we denote the direct limit of the sequence

$$
Q \rightarrow Q \times\{0\} \hookrightarrow Q \times Q \rightarrow Q \times Q \times\{0\} \hookrightarrow Q \times Q \times Q \ldots
$$

A closed subset $A$ of a space $X$ is called a Z-set in $X$, if the identity map of $X$ can be approximated (in the topology of uniform convergence) by maps whose image misses $A$.

\section{Hyperspaces of maX-Plus CONVEX POlyhedra}

The cone cone $(X)$ of a topological space $X$ is the quotient space $(X \times[0,1]) /(X \times\{0\})$. As usual, $\mathbb{R} P^{n}$ stands for the $n$-dimensional real projective space.

Proposition 2.1. The set $\operatorname{mpcc}_{2}\left(I^{n}\right)$ is homeomorphic to cone $\left(\mathbb{R} P^{n}\right) \times I^{n-1}$.

Proof. Let $\alpha: \exp _{2} I^{n} \rightarrow \operatorname{mpcc}_{2}\left(I^{n}\right)$ denote the map that assigns to every $A$ its max-plus convex hull. Then, clearly, $\alpha$ is a homeomorphism. The result follows from [18].

Proposition 2.2. Let $X$ be a compact max-plus convex subspace in a Euclidean space $\mathbb{R}^{k}$. Then the space $\operatorname{mpcc}_{n}(X)$ is an $A R$-space, for any $n \in \mathbb{N}$.

Proof. We proceed by induction. Clearly, $\operatorname{mpcc}_{n}(X)$ is homeomorphic to $X$ and therefore is an AR-space. Assume that we have already shown that $\operatorname{mpcc}_{n-1}(X)$ is an AR-space. Without loss of generality, one may assume that $X$ is a max-plus convex subset in a cube $J^{k}$, where $J$ is a closed segment in $\mathbb{R}$. Let $\alpha:\left(J^{k}\right)^{n} \rightarrow$ $\operatorname{mpcc}_{n}\left(J^{k}\right)$ be the map defined by the formula

$$
\alpha\left(x_{1}, \ldots, x_{n}\right)=h_{\mathrm{mp}}\left(\left\{x_{1}, \ldots, x_{n}\right\}\right)
$$

Note that the subspace $\alpha^{-1}\left(\operatorname{mpcc}_{n-1}(X)\right)$ of $\left(J^{k}\right)^{n}$ is determined by finitely many linear inequalities and therefore is an ANR-space. By [5], the adjunction space

$$
Y=\left(J^{k}\right)^{n} \cup_{\alpha \mid \alpha^{-1}\left(\operatorname{mpcc}_{n-1}(X)\right)} \alpha^{-1}\left(\operatorname{mpcc}_{n-1}(X)\right)
$$

is an ANR-space.

The $n$th symmetric group $S_{n}$ acts naturally on the space $Y$ and the orbit space of this action is exactly

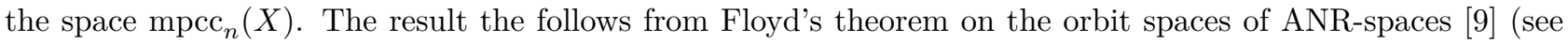
also [1]).

Let $p: I^{m} \rightarrow I^{n}$ denote the projection map, where $m \geq n$. This map induces the map of the hyperspaces $A \mapsto p(A): \operatorname{mpcc}_{i}\left(I^{m}\right) \rightarrow \operatorname{mpcc}_{i}\left(I^{n}\right)$; we denote it by $p_{i}^{*}$.

Proposition 2.3. Let $m>n \geq 2$. Then the map $p_{i}^{*}$ is open if and only if $i \in\{1,2\}$.

Proof. If $i=1$, then there is nothing to prove.

The map $p_{2}^{*}$ is open being homeomorphic to the map $A \mapsto p(A): \exp _{2} I^{m} \rightarrow \exp _{2} I^{n}$ (see the proof of Proposition 2.1). The latter map is known to be open (see, e.g., [15]).

Now suppose that $i \geq 3$. Let $A \in \operatorname{mpcc}_{i-1}\left(I^{n}\right) \backslash A \in \operatorname{mpcc}_{i-2}\left(I^{n}\right)$. Suppose that $A$ be the max-plus convex hull of a set $\left\{a_{1}, \ldots, a_{i-1}\right\}$. Without loss of generality, one may assume that there exists $B \in \operatorname{mpcc}_{i}\left(I^{m}\right) \backslash$ $\operatorname{mpcc}_{i-1}\left(I^{m}\right)$ with the property that $B \cap f^{-1}\left(a_{1}\right)$ is not a singleton.

One can also suppose that there is a sequence $\left(A_{j}\right)$ in $\operatorname{mpcc}_{i}\left(I^{n}\right) \backslash \operatorname{mpcc}_{i-1}\left(I^{n}\right)$ such that $A_{j}$ is the max-plus convex hull of the set $A \cup\left\{b_{j}\right\}$, where $\left(b_{j}\right)$ is a sequence in $I^{n}$ converging to $a_{2}$. 
Then, clearly, there is no sequence $\left(B_{j}\right)$ in $\operatorname{mpcc}_{i}\left(I^{m}\right)$ such that $\lim _{j \rightarrow \infty} B_{j}=A_{j}$ and $p_{i}^{*}\left(B_{j}\right)=A_{j}$ for every $j$. In turn, this implies that the map $p_{i}^{*}$ is not open.

Counterparts of the results of this section can be proved also for the max-min convexity.

\section{MaX-Plus CONVEX CONE}

A map $f: X \rightarrow Y$ of topological spaces is called soft [15] if, for every paracompact space $Z$, every its closed subset $A$, and every maps $\varphi: A \rightarrow X, \psi: Z \rightarrow Y$ with $\psi \mid A=\varphi$, there exists a map $\Phi: Z \rightarrow Y$ such that $f \Phi=\psi$ and $\Phi \mid A=\varphi$.

We define the max-convex cone of the cube $I^{\tau}$ as follows:

$$
\text { cone }_{\mathrm{mp}}\left(I^{\tau}\right)=\left\{\left(\left(x_{\alpha}\right), t\right) \mid t \in[0,1], x_{\alpha} \geq t \text { for all } \alpha\right\} \subset I^{\tau} \times I=I^{\tau+1} .
$$

Clearly, cone $\mathrm{mp}^{\tau}\left(I^{\tau}\right)$ is a max-plus convex subset in $I^{\tau+1}$.

Proposition 3.1. The hyperspace $\operatorname{mpcc}\left(\operatorname{cone}_{\mathrm{mp}}\left(I^{\omega}\right)\right)$ is homeomorphic to $I^{\omega}$.

Proof. We represent cone $\mathrm{mp}\left(I^{\omega}\right)$ as inverse limit $\lim _{(}\left(\right.$cone $\left._{\mathrm{mp}}\left(I^{m}\right), \pi_{m}\right)$, where the bonding maps $\pi_{m}$ are defined by the formula

Then

$$
\pi_{m}\left(\left(x_{1}, \ldots, x_{m+1}\right), t\right)=\left(\left(x_{1}, \ldots, x_{m}\right), t\right)
$$

$$
\operatorname{mpcc}\left(\operatorname{cone}_{\mathrm{mp}}\left(I^{\omega}\right)\right)=\lim _{\longleftarrow}\left(\operatorname{mpcc}\left(\operatorname{cone}_{\mathrm{mp}}\left(I^{m}\right)\right), \pi_{m}^{*}\right),
$$

where $\pi_{m}^{*}$ is the map defined as $A \mapsto \pi_{m}(A)$.

Similarly as in [2] one can show that the maps $\pi_{m}^{*}$ are open. Then, by applying selection theorem from [19] we conclude that these maps are soft and therefore the limit space $\operatorname{mpcc}\left(\operatorname{cone} e_{m p}\left(I^{\omega}\right)\right)$ is an absolute retract (see $[15])$.

Now, we are going to establish the DDP for the space $\operatorname{mpcc}\left(\operatorname{cone}_{\mathrm{mp}}\left(I^{\omega}\right)\right)$. Since this space is an AR-space, it is enough to prove that its identity map cam be approximated in the sense of uniform convergence by maps with disjoint images.

Let $\xi \in(0,1)$. Denote by $f_{m, \xi}, g_{m, \xi}:$ cone $_{\mathrm{mp}}\left(I^{\omega}\right) \rightarrow$ cone $_{\mathrm{mp}}\left(I^{\omega}\right)$ the maps acting by

$$
\left.\left.f_{m, \xi}\left(\left(x_{i}\right)_{i=1}^{\infty}, t\right)=\left(x_{1}, \ldots, x_{m}, 0, x_{m=2}, x_{m+3}, \ldots\right), \xi t\right), g_{m, \xi}\left(\left(x_{i}\right)_{i=1}^{\infty}, t\right)=\left(x_{1}, \ldots, x_{m}, \xi, x_{m=2}, x_{m+3}, \ldots\right), \xi t\right) .
$$

The maps $f_{m, \xi}$ and $g_{m, \xi}$ induce the maps $f_{m, \xi}^{*}$ and $g_{m, \xi}^{*}$ of the hyperspaces of the max-plus convex sets. Clearly, the images of the maps $f_{m, \xi}^{*}$ and $g_{m, \xi}^{*}$ are disjoint and, if $\xi$ is close enough to 1 and $m$ is large enough then $f_{m, \xi}^{*}$ and $g_{m, \xi}^{*}$ are close enough to the identity map of $\operatorname{mpcc}\left(\operatorname{cone}_{\mathrm{mp}}\left(I^{\omega}\right)\right)$.

The result now follows from Toruńczyk's characterization theorem for the Hilbert cube $Q$.

Remark that the notion of the max-plus convex cone can be defined also for all compact max-plus convex sets.

\section{FunCtion SPACES}

The notion of max-plus convex sets can be naturally extended to some functional spaces. We consider here a very special case of spaces of continuous functions on compact metric spaces.

Let $X$ be a compact metric space. By $C(X)$ we denote the Banach space of continuous functions endowed with the sup-norm.

A subset $A \subset C(X)$ is said to be max-plus convex if $t \odot x \oplus y \in A$, for every $x, y \in A$ and $t \in[-\infty, 0]$. By $\operatorname{mpcc}(C(X))$, we denote the hyperspace of the max-plus convex sets in $C(X)$. 
Given a subset $A \subset C(X)$, we denote by $h_{\mathrm{mp}}(A)$ the max-plus convex hull of $A$, i.e. the minimal max-plus convex set containing $A$.

Lemma 4.1. The max-plus convex hull of a finite set in $C(X)$ is compact.

Proof. Let $A \subset C(X)$ be finite and let $c=-\max \{\|x\| \mid x \in A\}$. It is easy to see that

$$
h_{\mathrm{mp}}(A)=\oplus\left\{t_{x} \odot x \mid x \in A, t_{x} \in[c, 0]\right\} .
$$

Therefore, $h_{\mathrm{mp}}(A)$ is the image of the compact set $[c, 0]^{A}$ under the map

$$
\left(t_{x}\right)_{x \in A} \mapsto \oplus\left\{t_{x} \odot x \mid x \in A\right\},
$$

i.e. is compact.

The proof of the following statement is immediate.

Lemma 4.2. Let $x_{i}, y_{i} \in A, i=1, \ldots, n$. If $\left\|x_{i}-y_{i}\right\|<\varepsilon$, for every $i=1, \ldots, n$, then

$$
\left\|\left(\oplus_{i=1}^{n} t_{i} \odot x_{i}\right)-\left(\oplus_{i=1}^{n} t_{i} \odot y_{i}\right)\right\|<\varepsilon,
$$

for any $t_{1}, \ldots, t_{n} \in \mathbb{R}$.

Lemma 4.3. For every precompact set $A \subset C(X)$, the set $h_{\mathrm{mp}}(A)$ is also precompact.

Proof. Given $\varepsilon>0$, find a finite $(\varepsilon / 2)$-net $B$ in $A$. Then $h_{\mathrm{mp}}(B)$ is compact and there is a finite $(\varepsilon / 2)$-net $B$ in $h_{\mathrm{mp}}(B)$.

Now, let $x \in h_{\mathrm{mp}}(A)$. Write $x=\oplus_{i=1}^{k} \alpha_{i} \odot a_{i}$, where $\alpha_{i} \leq 0, a_{i} \in A_{i}, i=1, \ldots, k, \oplus_{i=1}^{k} \alpha_{i}=0$. For every $i$, one can find $b_{i} \in B$ such that $\left\|a_{i}-b_{i}\right\|<\varepsilon / 2$. Let $y \oplus_{i=1}^{k} \alpha_{i} \odot b_{i}$, then by Lemma $4.2,\|x-y\|<\varepsilon / 2$. There exists $c \in C$ such that $\|c-y\|<\varepsilon / 2$. Finally, $\|x-c\|<\varepsilon$.

Lemma 4.4. The map

$$
A \mapsto \overline{h_{\mathrm{mp}}(A)}: \exp (C(X)) \rightarrow \operatorname{mpcc}(C(X))
$$

is nonexpanding.

Proof. Suppose that $d_{H}(A, B)<r$, where $A, B \in \exp (C(X))$ and $r>0$.

Let $\varepsilon>0$. Let $x \in \overline{h_{\mathrm{mp}}(A)}$, then there exists $y \in h_{\mathrm{mp}}(A)$ such that $\|x-y\|<\varepsilon$. Write $y=\oplus_{i=1}^{k} \alpha_{i} \odot a_{i}$, where $\alpha_{i} \leq 0, a_{i} \in A_{i}, i=1, \ldots, k, \oplus_{i=1}^{k} \alpha_{i}=0$. For every $i$, find $b_{i} \in B$ such that $\left\|a_{i}-b_{i}\right\|<r$. Then $z=\oplus_{i=1}^{k} \alpha_{i} \odot b_{i} \in \overline{h_{\mathrm{mp}}(B)}$ and $\|x-z\|<r+\varepsilon$.

One can similarly prove that, for any $x \in \overline{h_{\mathrm{mp}}(B)}$, there exists $z \in \overline{h_{\mathrm{mp}}(A)}$ such that $\|x-z\|<r+\varepsilon$. Therefore, $d_{H}\left(\overline{h_{\mathrm{mp}}(A)}, \overline{h_{\mathrm{mp}}(B)}\right)<r+\varepsilon$. Since $\varepsilon>0$, we are done.

Proposition 4.5. Let $X$ be a compact metrizable space. Then the space $\operatorname{mpcc}(C(X))$ is an $A R$-space.

Proof. One can proceed similarly as in the proof of Lemma 3.1 from [4]. Define a $c$-structure on the space $\operatorname{mpcc}(C(X))$ as follows: given any $A_{1}, \ldots, A_{n} \in \operatorname{mpcc}(C(X))$, let

$$
F\left(\left\{A_{1}, \ldots, A_{n}\right\}\right)=\left\{\bigoplus_{i=1}^{n} \alpha_{i} \odot A_{i} \mid \alpha_{1}, \ldots, \alpha_{n} \in[-\infty, 0], \bigoplus_{i=1}^{n} \alpha_{i}=0\right\} .
$$

The verification of the conditions from the definition of $c$-structure is straightforward.

We are going to show that every set of the form $F\left(\left\{A_{1}, \ldots, A_{n}\right\}\right)$ is contractible. Let $A=\oplus_{i=1}^{n} A_{i}$. Then $A \in F\left(\left\{A_{1}, \ldots, A_{n}\right\}\right)$. Define a map

$$
H: F\left(\left\{A_{1}, \ldots, A_{n}\right\}\right) \times[0,1] \rightarrow F\left(\left\{A_{1}, \ldots, A_{n}\right\}\right)
$$


by the formula:

$$
H(C, t)=C \oplus(\ln t) \odot A .
$$

Note that $H$ is well-defined, $H(C, 0)=C \oplus\{-\infty\}=C$ and $H(C, 1)=C \oplus 0 \odot A=A$, for every $C \in$ $F\left(\left\{A_{1}, \ldots, A_{n}\right\}\right)$. Thus, $H$ contracts the set $F\left(\left\{A_{1}, \ldots, A_{n}\right\}\right)$ to $A$.

The rest of the proof follows that of [4, Lemma 3.1].

Theorem 4.6. Let $X$ be an infinite compact metrizable space. Then the space $\operatorname{mpcc}(C(X))$ is homeomorphic to the separable Hilbert space $\ell^{2}$.

Proof. By $\left[8\right.$, Theorem E], the space $\exp (C(X))$ is homeomorphic to $\ell^{2}$. Clearly, the map

$$
A \mapsto \overline{h_{\mathrm{mp}}(A)}: \exp (C(X)) \rightarrow \operatorname{mpcc}(C(X))
$$

is a proper retraction. Now, we use Theorem 1.1 to conclude that mpcc $(C(X))$ is an $\ell^{2}$-manifold.

Since the space $\operatorname{mpcc}(C(X))$ is contractible, we are done.

\subsection{Non-separable case}

By $\ell^{2}(\kappa)$ we denote the Hilbert space of density $\kappa$. K. Koshino [12, Main Theorem] proved, in particular, that if a space $X$ is connected, locally connected, topologically complete, nowhere locally compact, and for each point $x \in X$, any neighborhood of $x$ in $X$ is of density $\kappa$, then $\exp X$ is homeomorphic to $\ell^{2}(\kappa)$.

Theorem 4.7. Let $X$ be a compact Hausdorff space of weight $\kappa$. Then the hyperspace $\operatorname{mpcc}(C(X))$ is homeomorphic to $\ell^{2}(\kappa)$.

Proof. The Banach space $C(X))$ is known to be of density $\kappa$. By Koshino's theorem stated above, $\exp C(X)$ is homeomorphic to $\ell^{2}(\kappa)$. Now, we remark that the methods applied in Section 4 work also in the non-separable case. Therefore, the hyperspace mpcc $(C(X))$ is also homeomorphic to $\ell^{2}(\kappa)$ as an AR-space which is dominated by $\ell^{2}(\kappa)$.

The notion of max-plus convex set can be naturally defined for the space $\mathbb{R}^{\infty}$.

Theorem 4.8. The hyperspace $\operatorname{mpcc}\left(\mathbb{R}^{\infty}\right)$ is homeomorphic to $Q^{\infty}$.

Proof. Since

$$
\operatorname{mpcc}\left(\mathbb{R}^{\infty}\right) \simeq \operatorname{mpcc}\left(\stackrel{\lim }{\longrightarrow}[-n, n]^{n}\right) \simeq \lim _{\longrightarrow} \operatorname{mpcc}\left([-n, n]^{n}\right),
$$

it suffices to prove that, for every natural $n$, the set $\operatorname{mpcc}\left([-n, n]^{n}\right)$ is a Z-set in the space $\operatorname{mpcc}\left([-n-1, n+1]^{n+1}\right)$. Indeed, for every $r>0$, the map

$$
A \mapsto \bar{O}_{r}(A): \operatorname{mpcc}\left([-n-1, n+1]^{n+1}\right) \rightarrow \operatorname{mpcc}\left([-n-1, n+1]^{n+1}\right)
$$

is a map which is $r$-close to the identity and misses $\operatorname{mpcc}\left([-n, n]^{n}\right)$. It follows from the Z-set unknotting theorem (see [7]) that the pair

$$
\left(\operatorname{mpcc}\left([-n-1, n+1]^{n+1}\right), \operatorname{mpcc}\left([-n, n]^{n}\right)\right)
$$

is homeomorphic to the pair $(Q \times Q, Q \times\{0\})$, which implies the result.

Counterparts of the results of this section can be proved also for the max-min convexity. 


\section{B-CONVEXITY}

Let $\mathbb{R}_{+}^{n}=\left\{x \in \mathbb{R}^{n} \mid x \geq 0\right\}$. A subset $C \subset \mathbb{R}^{n}$ is said to be $\mathbb{B}$-convex (see, e.g., [6]) if, for any $x, y \in C$ and $t \in[0,1], t x \oplus y \in C$. The map $j: \mathbb{R}^{n} \rightarrow \mathbb{R}_{+}^{n}, j\left(x_{1}, \ldots, x_{n}\right)=\left(e^{x_{1}}, \ldots, e^{x_{n}}\right)$, is an embedding which sends the max-plus convex sets in $\mathbb{R}^{n}$ to $\mathbb{B}$-convex sets in $\mathbb{R}_{++}^{n} \subset \mathbb{R}_{+}^{n}$ and such that the preimage of every $\mathbb{B}$-convex set is a max-plus convex set. Indeed, let $A \subset \mathbb{R}^{n}$ be a max-plus convex set and $j(x), j(y) \in j(A), t \in[0,1]$ (without loss of generality, we assume that $t \in(0,1])$. Then $t j(x) \oplus j(y)=j(\ln t \odot x \oplus y) \in j(A)$.

This remark allows us to prove for the hyperspace $\mathbb{B}-\operatorname{cc}\left(\mathbb{R}^{n}\right), n \geq 2$, of compact $\mathbb{B}$-convex subsets of $\mathbb{R}_{+}^{n}$ counterparts of results of [2].

One can extend the notion of $\mathbb{B}$-convex set over arbitrary vector lattice. To be specific, let $\ell_{+}^{2}$ denote the positive cone of the separable Hilbert space $\ell^{2}$. We say that a subset $B$ of $\ell_{+}^{2}$ is $\mathbb{B}$-convex if for all $x, y \in B$ and all $t \in[0,1]$ one has $t x \oplus y \in B$.

Theorem 5.1. The hyperspace $\mathbb{B}-c c\left(\ell_{+}^{2}\right)$ is homeomorphic to $\ell^{2}$.

Proof. Since $\ell_{+}^{2}$ is complete separable, connected, locally connected, nowhere locally compact metric space, $\exp \ell_{+}^{2}$ is homeomorphic to $\ell^{2}$, by [8, Theorem E]. The rest of the proof follows that of the main result of Section 4 .

\section{REMARKS AND OPEN QUESTIONS}

The following questions remain open.

1) Let $U$ be an open subset of $\mathbb{R}^{\infty}$. Is the hyperspace $\operatorname{mpcc}(U)$ homeomorphic to $U \times Q^{\infty}$ ?

2) The notion of $\mathbb{B}$-convex set can be formulated also for the space $\ell^{2}$ endowed with the bounded-weak* topology (denoted by $\left.\mathrm{bw}^{*}\right)$. Is the hyperspace $\operatorname{mpcc}\left(\ell^{2}, \mathrm{bw}^{*}\right)$ homeomorphic to $\left(\ell^{2}, \mathrm{bw}^{*}\right)$ ?

3 ) Is the hyperspace of all max-plus (max-min) polyhedra in $\mathbb{R}^{n}, n \geq 2$, homeomorphic to the pre-Hilbert space $\ell_{\mathrm{f}}^{2}=\left\{\left(x_{i}\right) \in \ell^{2} \mid x_{i}=0\right.$ for all but finitely many $\left.i\right\}$ ?

\section{REFERENCES}

[1] S.A. Antonyan,Retraction properties of an orbit space, Mathematics of the USSR-Sbornik(1990),65(2), 305-321.

[2] L.E. Bazylevych, Hyperspaces of max-plus convex compact sets, Mat.Zametki 84(5)(2008), 658-666 (in Russian).

[3] L. E. Bazylevych, On the hyperspace of max-min convex compact sets, Methods Funct. Anal. Topology Vol. 15 (2009), no. 4, $322-332$.

[4] L. Bazylevych, D. Repovš, M. Zarichnyi, Hyperspaces of max-plus convex subsets of powers of the real line. J. Math. Anal. Appl. 394 (2012), no. 2, 481-487.

[5] K. Borsuk, Theory of retracts, Monografie Matematyczne 44. PWN-Polish Scientific Publishers, Warszawa, 1967. - 251 p.

[6] W. Briec, C.D. Horvath, B-convexity, Optimization 53 (2004), 103-127.

[7] T. A. Chapman, Lectures on Hilbert Cube Manifolds, Conf. Board Math. Sci. Reg. Conf. Ser. Math. 28, Amer. Math. Soc., Providence, R.I., 1975.

[8] D.W. Curtis, Hyperspaces homeomorphic to Hilbert space, Proc. Amer. Math. Soc., 75 (1979), $126-130$.

[9] E. Floyd, Orbit spaces of finite transformation groups, Duke Math. J. 1953. V. 20, 563-567.

[10] S. Gaubert, R. Katz, Max-Plus Convex Geometry. In: Relations and Kleene Algebra in Computer Science, Volume 4136 of the series Lecture Notes in Computer Science, 192-206.

[11] C.D. Horvath, Contractibility and generalized convexity, J. Math. Anal. Appl. 156(2)(1991), 341-357.

[12] K. Koshino, On a hyperspace of compact subsets which is homeomorphic to a non-separable Hilbert space, arXiv:1508.05557.

[13] G.L. Litvinov, V.P. Maslov, and G.B. Shpiz, Idempotent functional analysis: an algebraic approach. Math. Notes, 69(5), 2001, 696-729.

[14] S. B. Nadler, Jr., J. Quinn and N. M. Stavrakos, Hyperspace of compact convex sets, Pacif. J. Math. 83(1979), $441-462$.

[15] E. V. Shchepin, Functors and uncountable powers of compacta, Uspekhi Mat. Nauk 31 (1981), 3-62.

[16] H. Toruńczyk, On CE-images of the Hilbert cube and characterization of Q-manifolds, Fund. math. 1980. T. 106, N 1. P. $31-40$.

[17] H. Toruńczyk, Characterizing Hilbert space topology, Fund. Math., 111 (1981), 247-262.

[18] C.H. Wagner, Symmetric, cyclic, and permutation products of manifolds, Rozprawy Matematyczne, T. 182, 1980,48 p.

[19] M. Zarichnyi, Michael selection theorem for max-plus compact convex sets, Topol. Proceedings, 31, $2007,677-681$. 